\title{
Semiconductor Nano-electronics for Quantum Information and Sensing
}

\author{
Dr. Jonathan Baugh \\ University of Waterloo, Canada
}

Nanostructured electronic devices allow us to control the way electrons and holes flow through a material, usually by imposing strong confinement in 1,2 or 3 dimensions. Top-down fabrication enables many device structures on various material platforms to be explored, yielding a rich array of physics in the quantum/nano regime. For example, confinement in all 3 dimensions produces a quantum dot, whose transport behaviour is dominated by strong electron-electron interaction and tunneling. Electrostatically-defined quantum dots in silicon can host long-lived electron spin quantum bits (qubits), providing a pathway for large-scale quantum computing analogous to conventional CMOS circuits. In addition to experimental work on few-electron quantum dots in silicon, we have proposed a scalable architecture based on a network of small nodes, and have performed simulations of its performance. In the realm of quantum sensing, we have developed suspended carbon nanotube electro-mechanical oscillators aimed at force detection of individual magnetic molecules. These devices combine quantum dot transport behaviour with mechanical motion, and can exhibit feedback self-driving effects. With these and other examples, I will give a perspective on the role of nanotechnology in future quantum technology development. 